\title{
A dieta com restrição de FODMAP reduz os sintomas no síndroma do intestino irritável? Uma revisão baseada na evidência
}

Paulo Diogo Terroso Mesquita Oliveira, ${ }^{1}$ Joana Estrela Felício Reis, ${ }^{1}$ Marisa Alexandra Sousa Reis, ${ }^{1}$ Sara Maria Santos Ferreira, ${ }^{1}$ André Lopes Albergaria Candelária²

\section{RESUMO}

Objetivos: Rever a evidência disponível sobre a eficácia da dieta com restrição de FODMAP (sigla inglesa para oligossacarídeos, dissacarídeos, monossacarídeos e poliois fermentáveis) no alívio dos sintomas gastrointestinais dos doentes com síndroma do intestino irritável (SII).

Métodos: Pesquisa de meta-análises, revisões sistemáticas e normas de orientação clínica na MEDLINE/PubMed, DARE, Trip DataBase, Evidence-based Medicine, Clinical Evidence, Web of Science, Canadian Medical Association Practice Guidelines, National Guidelines Clearing House e The Cochrane Library, publicados entre 2009 e 2017 na língua inglesa, utilizando os termos MeSH irritable bowel syndrome, signs and symptoms, diet therapy e o termo não MeSH 'FODMAPs'. Foi utilizada a escala Strenght of Recommendation Taxonomy (SORT) para atribuição de níveis de evidência e forças de recomendação.

Resultados: Depois de analisados 203 artigos resultantes da pesquisa foram incluídos 11 artigos: três meta-análises, três revisões sistemáticas e cinco normas de orientação clínica. Existe evidência de que a dieta com restrição em FODMAP (DRFODMAP) reduz a globalidade dos sintomas gastrointestinais dos doentes com SIl, com maior expressão na melhoria do bloating* e dor abdominais e nos subtipos de predomínio de diarreia e misto. Alguns estudos sugerem que a eficácia da dieta é mais evidente a partir da sexta semana. Esta dieta parece ser pelo menos tão eficaz no alívio sintomático quanto outras dietas convencionalmente recomendadas para o SII. Nas normas de orientação clínica analisadas, a DRFODMAP é considerada como uma opção terapêutica no SII, sobretudo quando o aconselhamento dietético tradicional não é eficaz.

Conclusão: Existe evidência para recomendar uma DRFODMAP como prova terapêutica em doentes com SIl com predomínio de quadro diarreico ou misto, sobretudo se bloating e dor abdominais presentes (força de recomendação B). São necessários mais estudos que suportem a eficácia e a segurança a longo prazo da dieta.

Palavras-chave: FODMAP; Síndroma do intestino irritável; Sinais e sintomas gastrointestinais; Terapia dietética.

\section{INTRODUÇÃO}

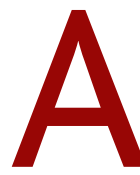

síndroma do intestino irritável (SII) é uma perturbação funcional de elevada prevalência, estimada entre os 10 e $20 \%$ nos países desenvolvidos, ${ }^{1-2}$ sendo mais frequente em mulheres e jovens adultos. ${ }^{3}$ Esta patologia representa um

1. USF Servir Saúde, ACeS Almada/Seixal.

2. USF Poente, ACES Almada/Seixal. encargo socioeconómico importante para os sistemas de saúde, tanto no elevado número de consultas médicas, requisição de exames complementares e tentativas de tratamento, como pelo absentismo e diminuição da qualidade de vida destes doentes. ${ }^{4}$ É o diagnóstico gastroenterológico mais comum nos cuidados de saúde primários, ${ }^{5}$ sendo responsável por $30 \%$ das referenciações à gastroenterologia pelos médicos de família. ${ }^{6}$

O SII engloba uma variedade de sintomas digestivos 


\begin{tabular}{|c|c|c|}
\hline Categoria & Alimentos com alto teor em FODMAP & $\begin{array}{l}\text { Alimentos alternativos com baixo teor em } \\
\text { FODMAP }\end{array}$ \\
\hline Vegetais & $\begin{array}{l}\text { Alcachofra, espargos, couve-flor, alho, cogumelos, } \\
\text { cebola, ervilhas. }\end{array}$ & $\begin{array}{l}\text { Beringela, curgete, cenoura, alface, batata, } \\
\text { tomate, abobrinha, feijão-verde, pimento. }\end{array}$ \\
\hline Frutas & $\begin{array}{l}\text { Maçã, sumo de maçã, cerejas, fruta desidratada, } \\
\text { nectarinas, pêssegos, pera, ameixas, melancia. }\end{array}$ & $\begin{array}{l}\text { Uvas, kiwi, tangerina, laranja, ananás, morangos, } \\
\text { meloa. }\end{array}$ \\
\hline Laticínios e derivados & $\begin{array}{l}\text { Leite de vaca, leite condensado, leite de soja } \\
\text { (que usa o grão integralmente), iogurte. }\end{array}$ & $\begin{array}{l}\text { Leite de amêndoa, queijo camembert, queijo } \\
\text { feta, queijo duro, leite sem lactose, leite soja } \\
\text { (que usa apenas a parte proteica). }\end{array}$ \\
\hline Proteínas & $\begin{array}{l}\text { Maioria das leguminosas, algumas carnes } \\
\text { processadas ou marinadas, carne de aves, marisco. }\end{array}$ & $\begin{array}{l}\text { Ovos, tofu, carne de aves e marisco bem } \\
\text { cozinhados, tempeh. }\end{array}$ \\
\hline Pão e cereais & $\begin{array}{l}\text { Cereais/pães que contenham centeio, trigo, } \\
\text { cevada, bolachas. }\end{array}$ & $\begin{array}{l}\text { Cereais/pães que contenham milho/espelta, } \\
\text { quinoa, arroz. }\end{array}$ \\
\hline Açúcar/adoçantes & $\begin{array}{l}\text { Xarope com fructose, mel, sorbitol, manitol, } \\
\text { isomalte. }\end{array}$ & $\begin{array}{l}\text { Chocolate negro, xarope de maple, xarope de } \\
\text { malte de arroz, adoçantes que contenham } \\
\text { sacarose, glicose. }\end{array}$ \\
\hline Frutos secos e sementes & Pistachio, caju. & $\begin{array}{l}\text { Noz de macadâmia, amendoins, sementes de } \\
\text { abóbora, nozes. }\end{array}$ \\
\hline
\end{tabular}

Nota: Adaptado pelos autores a partir da versão original disponível (https://www.monashfodmap.com/about-fodmap-and-ibs/high-and-low-fodmapfoods/).

intermitentes. Atualmente define-se, segundo os critérios de Roma IV, por dor abdominal recorrente (pelo menos um dia por semana durante os últimos três meses) associada a dois ou mais dos seguintes sintomas: 1 ) relacionada com a defecação; 2) associada a alteração da frequência das fezes; ou 3) associada a alteração forma/aparência das fezes. ${ }^{7}$ Pode ainda ser subclassificado, de acordo com a consistência das fezes e segundo a escala de Bristol, em quatro subtipos: SII com predomínio de diarreia (SII-D); SII com predomínio de obstipação (SII-O); SII misto (SII-M); e SII inclassificável (SII-I). ${ }^{\text {? }}$

A sua etiologia é multifatorial ejulga-se que possam estar envolvidos fatores como a hipersensibilidade visceral, a alteração da motilidade e da flora do trato gastrointestinal, a inflamação intestinal, a suscetibilidade genética, fatores ambientais e perturbações do foro psicossocial. ${ }^{8}$ O tratamento da doença torna-se, por este motivo, um desafio e baseia-se numa combinação de estratégias que incluem terapêutica farmacológica, dietas restritivas, probióticos e terapia cognitivo-comportamental. ${ }^{9}$
As medidas não farmacológicas são a primeira opção de tratamento, sendo a dieta considerada a intervenção mais eficaz. ${ }^{10}$ Neste contexto, em 2009, uma equipa de investigadores da Universidade Australiana de Monash desenvolveu uma dieta restritiva em FODMAP (sigla inglesa para oligossacarídeos, dissacarídeos, monossacarídeos e poliois fermentáveis). Os FODMAP são hidratos de carbono de cadeia curta pouco absorvidos no intestino, onde são osmoticamente ativos e rapidamente fermentados, agravando os sintomas no SII. Estes encontram-se em alimentos que contenham frutanos, galactanos, lactose, frutose, sorbitol ou manitol (Quadro I). ${ }^{11}$ A dieta com restrição de FODMAP tem sido considerada uma abordagem promissora na gestão dos sintomas desta doença, mas a sua recomendação parece ainda não ser consensual.

Assim, esta revisão baseada na evidência pretende determinar se a dieta com restrição de FODMAP (DRFPODMAP) é eficaz na redução dos sintomas nos doentes com SII. 


\begin{tabular}{|c|c|c|}
\hline Referência (ano) & Recomendação & $\begin{array}{c}\text { Força de } \\
\text { Recomendação }\end{array}$ \\
\hline $\begin{array}{l}\text { American College of } \\
\text { Gastroenterology }(2014)^{14}\end{array}$ & A DRFODMAP pode melhorar os sintomas nos doentes com SII. & C \\
\hline $\begin{array}{l}\text { World Gastroenterology } \\
\text { Organisation }(2015)^{15}\end{array}$ & $\begin{array}{l}\text { Recomenda a DRFODMAP por melhorar a dor abdominal, bloating e o padrão } \\
\text { de dejeções. Não é claro se esta é benéfica para todos os subtipos de SII. }\end{array}$ & C \\
\hline $\begin{array}{l}\text { British Dietetic Association } \\
(2016)^{16}\end{array}$ & $\begin{array}{l}\text { Recomenda a DRFODMAP por 3-6 semanas, com acompanhamento por } \\
\text { nutricionista, como aconselhamento dietético de segunda linha. }\end{array}$ & B \\
\hline $\begin{array}{l}\text { Canadian Association of } \\
\text { Gastroenterology }(2016)^{17}\end{array}$ & Recomenda a DRFOMAP como opção terapêutica nos subtipos SII-D e SII-M. & C \\
\hline NICE $(2017)^{18}$ & $\begin{array}{l}\text { Recomenda a DRFODMAP com acompanhamento por um profissional de saúde } \\
\text { especializado, se alteração do estilo de vida e aconselhamento dietético geral } \\
\text { não foram eficazes no controlo sintomático. }\end{array}$ & C \\
\hline
\end{tabular}

\section{MÉTODOS}

Foi realizada uma pesquisa bibliográfica ${ }^{12} \mathrm{em}$ janeiro de 2018 de meta-análises (MA), revisões sistemáticas (RS) e normas de orientação clínica (NOC) indexadas na MEDLINE/PubMed, DARE, Trip DataBase, Evidence-based Medicine, Clinical Evidence, Web of Science, Canadian Medical Association Practice Guidelines, National Guidelines Clearing House e The Cochrane Library, publicadas entre 2009 e 2017 na língua inglesa. Foram utilizados os termos MeSH irritable bowel syndrome, signs and symptoms e diet therapye o termo não MeSH 'FODMAPs'. Os critérios utilizados para a inclusão de artigos nesta revisão foram definidos segundo o modelo PICO: População - doentes com SII de todas as idades; Intervenção - dieta com restrição em FODMAP; Comparador - outras dietas; Outcome-melhoria dos sintomas GI. A pesquisa e a seleção dos artigos foram realizadas por cinco investigadores e a sua inclusão na revisão foi discutida e decidida unanimemente. A avaliação da qualidade metodológica dos artigos foi debatida entre todos os autores e apresentada de forma descritiva, tendo em conta as características da amostra, da intervenção e do comparador, o método de aleatorização e ocultação, o tempo de seguimento e a medição da adesão e dos resultados. Foi utilizada a escala Strenght of Recommendation Taxonomy (SORT), da American Academy of Family Physicians, ${ }^{13}$ para atribuição de níveis de evidência (NE) e forças de recomendação (FR).

\section{RESULTADOS}

Da pesquisa inicial foram obtidos 203 artigos, dos quais onze cumpriram os critérios de inclusão (três MA, três RS e cinco NOC). O processo de seleção dos artigos encontra-se representado na Figura 1.

A descrição resumida dos artigos incluídos nesta revisão encontra-se na Quadro II (NOC), Quadro III (MA) e Quadro IV (RS). As limitações dos estudos incluídos são discutidas no final da secção dos resultados.

\section{Normas de orientação clínica}

A American College of Gastroenterologyconsidera que a DRFODMAP pode ter um papel promissor no controlo dos sintomas do SII, embora considere que a evidência atual é ainda limitada (estudos com baixa qualidade) e que as suas indicações exatas estão ainda por definir (FR C).${ }^{14} \mathrm{~A}$ World Gastroenterology Organisation recomenda a dieta a doentes com dor abdominal, bloating* e alteração do padrão das dejeções, embora não considere claro se esta é benéfica para todos os subtipos de SII (FR C).$^{15}$ Ressalva ainda que a sua segurança e benefícios a longo prazo estão ainda por

\footnotetext{
*Apesar de frequentemente os termos bloating e distensão abdominal serem usados como equivalentes, o bloating corresponde à sensação subjetiva (sintoma) de «inchaço» abdominal, enquanto a distensão abdominal é um sinal objetivamente quantificável (pois implica protusão abdominal). Dada a inexistência de um termo técnico para bloating na língua portuguesa, os autores optaram por manter a sua nomenclatura na língua inglesa (nota dos autores).
} 


\begin{tabular}{|c|c|c|c|c|}
\hline $\begin{array}{l}\text { Referência } \\
\text { (ano) }\end{array}$ & $\begin{array}{l}\text { Estudos incluídos } \\
\text { e dimensão } \\
\text { da amostra }\end{array}$ & Comparador & Resultados & NE \\
\hline $\begin{array}{l}\text { Marsh et al. } \\
(2015)^{19}\end{array}$ & $\begin{array}{l}6 \text { ECCA } \\
N=354\end{array}$ & $\begin{array}{l}\text { Dieta mediterrânica; } \\
\text { dieta tradicionalmente } \\
\text { recomendada para SII } \\
\text { Follow-up: } \\
\text { 3-6 semanas }\end{array}$ & $\begin{array}{l}\text { A DRFODMAP demonstrou: } \\
\text { - melhoria na escala de sintomas IBS-SSS (OR=0,44; IC } 95 \% \text {, } \\
\left.0,25-0,76 ; I^{2}=35,52 ; p=0,00\right) \\
\text { - redução significativa da gravidade dos seguintes sintomas: } \\
\left.\text { dor abdominal (OR=1,81; IC } 95 \%, 1,13-2,88 ; I^{2}=0,00 ; p=0,56\right) \text {, } \\
\left.\text { bloating (OR=1,75; IC05\%, } 1,07-2,87 ; I^{2}=0,00 ; p=0,45\right) \text { e } \\
\text { globalidades dos sintomas (OR=1,81; IC } 95 \%, 1,11-2,95 ; \\
\left.I^{2}=0,00 ; p=0,4\right) \\
\text { - melhoria da qualidade de vida (OR=1,84; IC } 95 \%, 1,12-3,03 ; \\
\left.\mathrm{i}^{2}=0,00 ; p=0,39\right)\end{array}$ & 2 \\
\hline $\begin{array}{l}\text { Varjú et al } \\
(2017)^{20}\end{array}$ & $\begin{array}{l}5 \text { ECCA; } \\
1 \text { estudo } \\
\text { cross-over; } \\
1 \text { estudo } \\
\text { prospetivo } \\
\text { controlado; } \\
3 \text { estudos } \\
\text { prospetivos não } \\
\text { controlados. } \\
N=489\end{array}$ & $\begin{array}{l}\text { Dieta habitualmente } \\
\text { aconselhada para SII } \\
\text { (segundo as NOC) } \\
\text { Follow-up: } \\
3 \text { a } 13 \text { semanas }\end{array}$ & $\begin{array}{l}\text { - Ambas as intervenções são eficazes na redução dos sintomas } \\
\text { e melhoria da qualidade de vida dos doentes com SII } \\
\text { [diferença média da escala IBS-SSS entre pré e pós } \\
\text { intervenção, no grupo controlo: }-59,8 \text { (IC95\%, }-108,9 \text { a }-10,7 ; \\
P=0,017) \text { e no grupo intervenção }[-105,3 \text { (IC95\%, }-140,8 \text { a } \\
-69,9 ; p=0,000) \text { ] } \\
\text { - A DRFODMAP é mais eficaz na redução dos sintomas do SII } \\
\text { do que a dieta tradicionalmente recomendada nas NOC } \\
\text { [diferença média na escala de IBS-SSS entre grupo controlo e } \\
\text { grupo intervenção, pré-intervenção: }-8,7(\text { IC } 95 \%,-40,0 \text { a } \\
+22,7 ; p=0,588) \text { e pós-intervenção }[+51,5(I C 95 \%,+18,9 \text { a } \\
+84,2 ; p=0,002)]\end{array}$ & 2 \\
\hline $\begin{array}{l}\text { Altobelli et } \\
\text { al }(2017)^{21}\end{array}$ & $\begin{array}{l}6 \text { ECCA; } \\
6 \text { estudos coorte. } \\
N=589\end{array}$ & $\begin{array}{l}\text { Dieta tradicional; } \\
\text { dieta com teor médio/ } \\
\text { /elevado de FODMAP } \\
\text { Follow-up: } \\
3 \text { semanas a } 9 \text { meses }\end{array}$ & $\begin{array}{l}\text { - DRFODMAP é mais eficaz que dieta tradicional na redução } \\
\text { de dor abdominal, bloating e frequência de dejeções [dor } \\
\text { abdominal: OR=0,44 (IC95\%,0,2-0,79; } p=0,006) ; \text { bloating: } \\
\text { OR=0,32 (IC95\%, 0,15-0,61; } p<0,0001) \text {; diarreia: ES -0,54 } \\
\text { (IC95\%, -0,83 a -0,24; } p<0,0001) \text { ] } \\
\text { - DRFODMAP, comparada a dieta com médio/elevado teor } \\
\text { em FODMAP, demonstrou redução significativa de dor } \\
\text { abdominal e bloating [dor abdominal: OR=-0,59 (IC95\%, } \\
-0,76 \text { a -0,42; } p<0,0001) ; \text { bloating: OR=-0,64 (IC95\%, 0,82 a } \\
-0,46 ; p<0,0001) \text { ]. }\end{array}$ & 2 \\
\hline
\end{tabular}

determinar. A British Dietetic Association recomenda a DRFODMAP como aconselhamento dietético de segunda linha, por 3-6 semanas, ressaltando que a sua eficácia não está comprovada se não existir acompanhamento dietético por nutricionista (FR B).${ }^{16} \mathrm{~A}$ Canadian Association of Gastroenterology recomenda a DRFODMAP como parte integrante do tratamento do SII com predomínio de diarreia e misto (FR C) ${ }^{17} \mathrm{O}$ National Institute for Health and Care Excellence (NICE) recomenda a DRFODMAP, se as medidas dietéticas gerais não forem eficazes no alívio dos sintomas, desde que acompanhada por um profissional de saúde especializado (FR C). ${ }^{18}$

\section{Meta-análises}

A MA de Marsh e colaboradores teve como objetivo avaliar a eficácia da DRFODMAP na redução dos sintomas associados ao SII. Esta MA incluiu seis ensaios clínicos controlados aleatorizados (ECCA), 


\begin{tabular}{|c|c|c|c|c|}
\hline Referência (ano) & $\begin{array}{l}\text { Estudos incluídos } \\
\text { e amostra }\end{array}$ & Comparador & Resultados & NE \\
\hline Rao et al $(2015)^{22}$ & $\begin{array}{l}3 \text { ECCA; } \\
2 \text { estudos } \\
\text { observacionais } \\
\text { prospetivos; } \\
1 \text { estudo } \\
\text { observacional } \\
\text { retrospetivo } \\
N=381 \\
\text { (257 DRFODMAP/ } \\
\text { /124 controlos) }\end{array}$ & $\begin{array}{l}\text { Dieta sem restrições; } \\
\text { dieta recomendada } \\
\text { pelo NICE; } \\
\text { dieta habitualmente } \\
\text { aconselhada para SII } \\
\text { (segundo as NOC) } \\
\text { Follow-up: } \\
2 \text { dias a } 16 \text { meses }\end{array}$ & $\begin{array}{l}\text { Melhoria estatisticamente significativa da globalidade } \\
\text { dos sintomas, a partir da primeira semana e maior } \\
\text { eficácia a partir da sexta semana. } \\
\text { Resultados inconsistentes relativamente aos benefícios } \\
\text { em cada subtipo. }\end{array}$ & 2 \\
\hline $\begin{array}{l}\text { Wong et al } \\
(2016)^{23}\end{array}$ & $\begin{array}{l}1 \mathrm{MA} ; 1 \mathrm{RS} ; \\
9 \text { ECCA; } \\
2 \text { estudos } \\
\text { comparativos; } \\
3 \text { estudos } \\
\text { observacionais } \\
\text { prospetivos; } \\
1 \text { caso de estudo; } \\
3 \text { NOC } \\
N=690\end{array}$ & $\begin{array}{l}\text { Dieta habitualmente } \\
\text { aconselhada para SII } \\
\text { (segundo as NOC); } \\
\text { dieta sem restrições } \\
\text { Follow-up: } \\
2 \text { dias a } 17 \text { meses }\end{array}$ & $\begin{array}{l}\text { Melhoria significativa do bloating na maioria dos estudos. } \\
\text { Um estudo não demonstrou melhoria do bloating, apesar } \\
\text { de revelar melhoria da globalidade dos sintomas. }{ }^{26} \\
\text { Redução dos sintomas desde } 2-8 \text { semanas, mais evidente } \\
\text { a partir da sexta semana. }\end{array}$ & 2 \\
\hline $\begin{array}{l}\text { Mckenzie et al } \\
(2016)^{16}\end{array}$ & $\begin{array}{l}5 \text { ECCA } \\
N=438\end{array}$ & $\begin{array}{l}\text { Dieta aconselhada } \\
\text { pela NICE; } \\
\text { dieta sem restrições } \\
\text { Follow-up: } \\
3 \text { a } 6 \text { semanas }\end{array}$ & $\begin{array}{l}\text { Redução estatisticamente significativa da globalidade } \\
\text { dos sintomas, quando cumprida por um período de } 3 \text { a } 6 \\
\text { semanas. } \\
\text { Resultados inconsistentes relativamente aos benefícios } \\
\text { em cada subtipo. }\end{array}$ & 2 \\
\hline
\end{tabular}

englobando 354 doentes com SII (segundo os critérios de Roma III). Foi analisado o efeito da dieta com restrição de FODMAP comparativamente a dieta sem restrições ou dieta tradicionalmente recomendada para SII, com duração entre três a seis semanas, através da escala de sintomas Irritable Bowel Syndrome - Symptoms Severity Score (IBS-SSS) e da escala de qualidade de vida Irritable Bowel Syndrome Quality of Life Score (IBS-QOL). Esta dieta demonstrou melhoria na escala de sintomas e de qualidade de vida nos doentes com SII. Os estudos incluídos nesta MA têm qualidade metodológica heterogénea: quatro apresentam ocultação única; dois não fornecem informação sobre a técnica de aleatorização nem dados sobre abandono do estudo. Assim, foi-lhe atribuído o nível de evidência (NE) 2.

A MA de Varjú e colaboradores analisou a eficácia terapêutica da DRFODMAP na redução dos sintomas e na melhoria da qualidade de vida dos doentes com SII, em comparação com a dieta recomendada nas NOC mais atuais, através da escala IBS-SSS. Foram incluídos cinco ECCA, um estudo cross-over, um estudo prospetivo controlado e três estudos prospetivos não controlados, tendo sido analisados 489 doentes com SII (segundo critérios de Roma II, III, IV ou critérios NICE). A duração dos estudos varia entre três e treze semanas. Os autores concluem que tanto a dieta tradicionalmente 


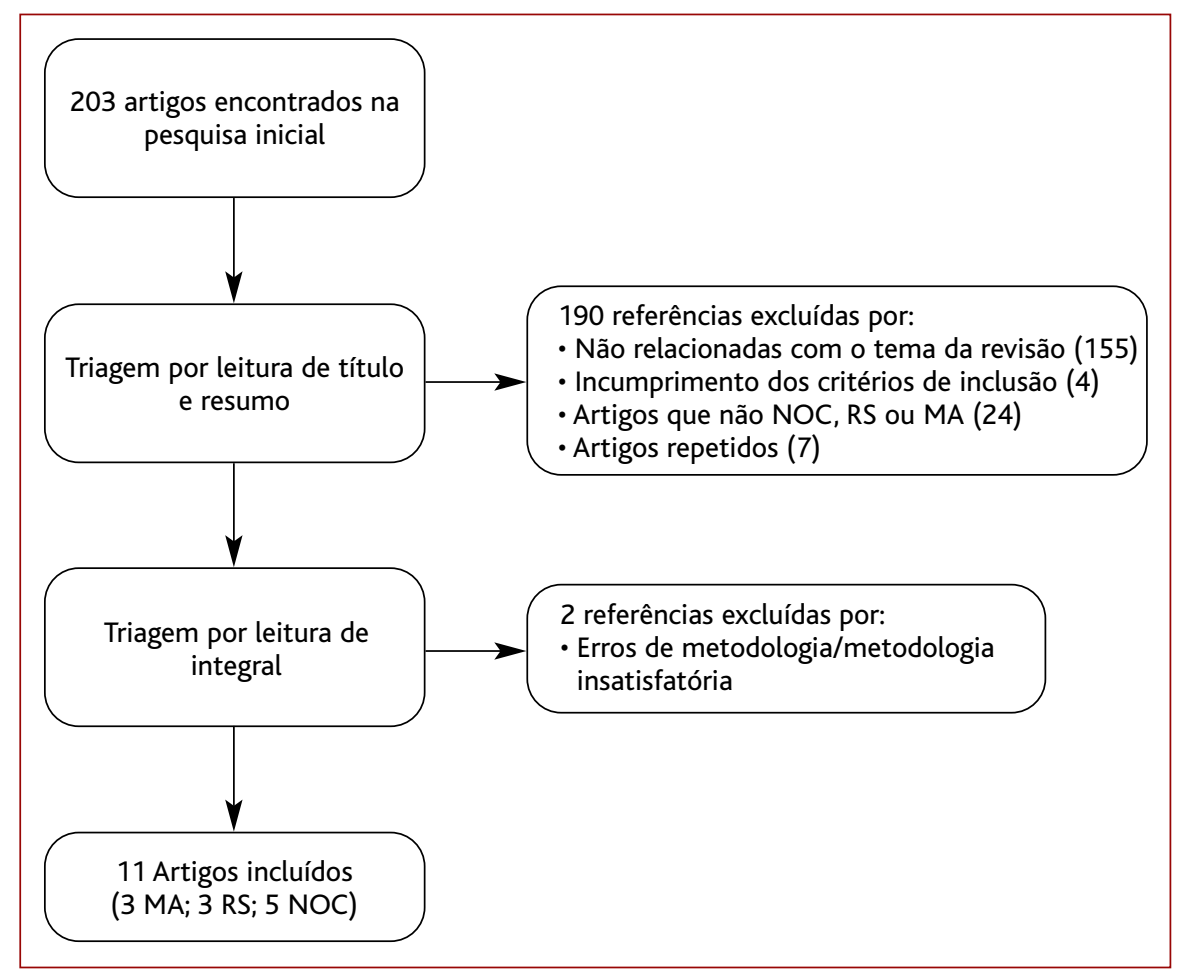

Figura 1. Fluxograma de seleção de artigos.

recomendada nas NOC para SII como a DRFODMAP são eficazes na redução dos sintomas e melhoria da qualidade de vida dos doentes com SII; porém, a DRFODMAP demonstrou eficácia superior. Um dos ECCA incluídos não apresenta descrição metodológica; os restantes ensaios controlados apresentam qualidade metodológica aceitável. Foi atribuído a esta MA um NE 2.

A MA de Altobelli e colaboradores comparou os efeitos da DRFODMAP com dietas tradicionais e dietas com teor médio a elevado em FODMAP na redução dos sintomas de diarreia, dor abdominal e bloating. Esta MA inclui 12 estudos (seis ECCA e seis estudos coorte), nos quais foram incluídos 589 doentes adultos com diagnóstico de SII (segundo critérios de Roma III). A duração dos estudos varia entre três semanas e nove meses. Foi demonstrado que a DRFODMAP, quando comparada com a dieta tradicional e dietas com maior teor em FODMAP, reduz significativamente os sintomas funcionais de SII, nomeadamente dor abdominal, bloatinge diarreia. Dos ECCA incluídos nesta MA dois apresentam ocultação única; nos restantes não foi realiza- da ocultação da intervenção. Um dos ECCA não fornece dados sobre abandono do estudo. Em dois estudos coorte, a percentagem de doentes que completaram o estudo foi insatisfatória. Por estes motivos, foi atribuído a esta MA um NE 2.

\section{Revisões sistemáticas}

A RS de Rao e colaboradores teve como objetivo principal avaliar a eficácia de DRFODMAP no tratamento dos sintomas de SII. Foram avaliados três ECCA e três estudos não aleatorizados, incluindo um total de 381 doentes com SII, segundo critérios Roma III ou NICE. Foi analisado o efeito da DRFODMAP nos sintomas deSII em comparação com dietas sem restrições, dietas com elevado teor em FODMAP, dietas tradicionalmente aconselhadas para SII e a dieta recomendada pela NICE, cumpridas por um período entre dois dias e 16 meses. Foram usadas as escalas IBSS, IBS-QOL e a Escala Visual Analógica (EVA). Todos os estudos incluídos demonstraram melhoria estatisticamente significativa da globalidade dos sintomas em doentes com DRFODMAP. Não se demonstrou benefício relativamente à diarreia e obstipação quando comparada com dieta recomendada pela NICE. De referir que um ECCA demonstrou uma melhoria sintomática significativa para o subtipo SII-D, mas não para SII-O ( $\nu$ s dieta sem restrições). ${ }^{24} \mathrm{Um}$ outro ECCA demonstrou melhoria semelhante para os subtipos SII-D e SII-O (FODMAP vs dietas com elevado teor em FODMAP) ${ }^{25}$ A qualidade dos estudos é heterogénea, sendo que os processos de aleatorização não são especificados; dois ECCA apresentam ocultação única e outro foi realizado sem ocultação. Os autores não procederam à avaliação do risco de viés dos estudos observacionais incluídos. Foi atribuído a esta RS um NE 2.

A RS de Wong avaliou a evidência de eficácia da DRFODMAP no alívio sintomático da sensação de bloating em pacientes com SII. Este artigo inclui uma 
MA, uma RS, nove ECCA, dois estudos comparativos não aleatorizados, três estudos observacionais prospetivos, um caso de estudo e três NOC. A população estudada inclui 690 doentes com SII, segundo critérios de Roma III ou NICE. É analisado o efeito da DRFODMAP em comparação a dietas sem restrições ou dieta tradicionalmente recomendada para SII, com duração entre dois dias a 17 meses, utilizando as escalas de sintomas IBS-SSS e EVA.Verificou-se uma redução significativa da gravidade de bloatinge score IBS-SSS em pacientes com SII em 14 dos 16 estudos incluídos (dois ECCA não demonstram benefício). Um estudo não demonstrou melhoria do bloating, apesar de revelar melhoria sintomática global. ${ }^{26}$ Os efeitos da intervenção nos sintomas de SII ocorrem após duas a oito semanas, sendo a melhoria sintomática mais evidente após a sexta semana. Um ECCA demonstrou que a intensidade dos sintomas de bloating é proporcional à quantidade de FODMAP ingeridos. Os ECCA revistos apresentam um risco de viés baixo a moderado; três das nove ECCA apresentam ocultação da alocação. No que respeita aos estudos não aleatorizados, os autores não procederam à sua avaliação crítica. Foi atribuído a esta RS um NE de 2.

Na RS de McKenzie e colaboradores, a DRFODMAP foi comparada com a dieta recomendada pela NICE e dietas sem restrições. Esta RS inclui cinco ECCA, no conjunto dos quais foram incluídos 438 doentes com SII (segundo os critérios Manning, Roma I, II ou III ou diagnosticado pelo clínico). Os autores concluem que a DRFODMAP reduz a globalidade dos sintomas funcionais, desde a terceira à sexta semana. Dois ECCA demonstraram melhoria sintomática semelhante para os subtipos SII-D e SIIO. ${ }^{25,27}$ Outro ECCA revelou uma melhoria significativa para o subtipo SII-D, mas não para o SII-O. ${ }^{24}$ Um estudo demonstrou eficácia semelhante na redução de sintomas à das recomendações dietéticas NICE. ${ }^{27}$ Os autores identificam como possível explicação o facto de o aporte de FODMAP em ambos os braços do estudo ter sido semelhante. Quanto à qualidade metodológica dos estudos incluídos, os autores avaliaram o risco de viés como alto em três estudos, baixo num estudo e pouco claro noutro estudo. Foi atribuído a esta RS o NE 2.

\section{Limitações}

Quanto às limitações das MA e RS incluídas nesta revisão são de referir: o número reduzido de estudos pri- mários e a sua qualidade metodológica (pequeno número de ECCA com ocultação por oposição a estudos observacionais com maior risco de viés); a heterogeneidade das intervenções (teor em FODMAP variável ou não especificado; níveis de adesão à dieta não quantificados); a heterogeneidade do comparador (desde dietas sem restrições ou com elevados teor em FODMAP a dietas recomendadas em NOC); a curta duração dos estudos incluídos não permite determinar a segurança e benefícios a longo prazo desta dieta; e a ausência de análise por subtipos de SII na maioria dos estudos incluídos limita as conclusões acerca de quais os subtipos que terão maior benefício com a DRFODMAP. As amostras dos estudos primários incluídos nesta revisão são suficientemente uniformes para permitir a comparação dos resultados (critérios de diagnóstico de SII, adultos, maioria do género feminino). Os participantes nos estudos primários têm características diversas (idade, subtipos de SII, comorbilidades, estatuto socioeconómico, hábitos alimentares, etc.), o que possibilita a aplicação dos resultados da revisão aos doentes que frequentam os cuidados de saúde primários.

De referir que nas MA e RS incluídas existe sobreposição de 16 em 33 estudos primários, com menor expressão nas MA (apenas quatro em 18 estudos primários). Embora a considerável sobreposição de estudos primários seja uma limitação na interpretação dos resultados, tendo em conta que a maioria dos estudos sobrepostos relata resultados favoráveis à dieta FODMAP, ressalve-se que estes são também os estudos com maior qualidade metodológica.

\section{CONCLUSÕES}

Os diversos estudos e recomendações demonstram que a dieta com restrição em FODMAP se associa a redução dos sintomas no SII e a ganho de qualidade de vida, com maior expressão na melhoria do bloating e dor abdominal e nos subtipos de predomínio diarreico e misto. Alguns estudos sugerem que a eficácia da dieta é superior a partir da sexta semana. É aconselhável um acompanhamento nutricional especializado, de forma a evitar dietas demasiado restritivas e défices nutricionais. Esta dieta parece ser pelo menos tão eficaz no alívio sintomático quanto outras dietas convencionalmente recomendadas para o SII. 
Existe evidência para recomendar uma dieta com restrição de FODMAP como prova terapêutica em doentes com SII com predomínio de quadro diarreico ou misto, sobretudo se presentes bloating e dor abdominal (força de recomendação B). São necessários mais estudos que suportem a eficácia e segurança a longo prazo da dieta e nos outros tipos de SII.

Considera-se que a DRFODMAP é uma opção válida para a gestão do SII, permitindo um melhor controlo dos sintomas e ganhos na qualidade de vida do doente, sem efeitos adversos significativos, resultando numa menor procura de cuidados de saúde e encargos para o Serviço Nacional de Saúde.

\section{AGRADECIMENTOS}

Agradecemos aos nossos orientadores de formação: Dra. Isabel Alves, Dra. Raquel Eça, Dr. Rui Capote e Dra. Célia Santos Silva.

\section{REFERÊNCIAS BIBLIOGRÁFICAS}

1. Longstreth GF, Thompson WG, Chey WD, Houghton LA, Mearin F, Spiller RC. Functional bowel disorders. Gastroenterology. 2006;130(5):148091.

2. Lovell RM, Ford AC. Global prevalence of and risk factors for irritable bowel syndrome: a meta-analysis. Clin Gastroenterol Hepatol. 2012;10 (7):712-21.e4.

3. Bharadwaj S, Barber MD, Graff LA, Shen B. Symptomatology of irritable bowel syndrome and inflammatory bowel disease during the menstrual cycle. Gastroenterol Rep. 2015;3(3):185-93.

4. Perry AF, Dellon ES, Lund J, Crockett SD, MCGowan CE, Bulsiewicz WJ. Burden of gastrointestinal disease in the United States: 2012 update. Gastroenterology. 2012;143(5):1179-87.e3.

5. Freitas DS. Síndrome do cólon irritável: estado da arte. Rev Port Coloproctol. 2004;1(1):53-69.

6. Pires E. Síndrome do Intestino Irritável. Rev Zen Energy. 2017;9(9):601.

7. Lacy BE, Patel NK. Rome criteria and a diagnostic approach to irritable bowel syndrome. J Clin Med. 2017;6(11):99.

8. Portincasa P, Bonfrate L, de Bari O, Lembo A, Ballou S. Irritable bowel syndrome and diet. Gastroenterol Rep. 2017;5(1):11-9.

9. Andrade V, Fonseca TN, Gouveia AC, Kobayashi TG, Leite RGS, Mattar RA et al. Dieta restrita de FODMAPs como opção terapêutica na síndrome do intestino irritável: revisão sistemática [FODMAPs restricted diet as a treatment option in irritable bowel syndrome: systematic review]. GED Gastroenterol Endosc Dig. 2014;34(1):34-41. Portuguese

10. Saha L. Irritable bowel syndrome: pathogenesis, diagnosis, treatment and evidence-based medicine. World J Gastroenterol. 2014;20(22): 6759-73.

11. Monash University. High and low FODMAP foods: a sample food list from the FODMAP experts [homepage]. Monash University; 2019. Available from: https://www.monashfodmap.com/about-fodmap-andibs/high-and-low-fodmap-foods/
12. Braga R, Melo M. Como fazer uma revisão baseada na evidência [How to make na evidence-based clinical review article]. Rev Port Clin Geral. 2009;25(6):660-6. Portuguese

13. Ebell MH, Siwek J, Weiss BD, Woolf SH, Susman J, Ewigman B, et al. Strength of Recommendation Taxonomy (SORT): a patient-centered approach to grading evidence in the medical literature. Am Fam Physician. 2004;69(3):548-56.

14. Ford AC, Moayyedi P, Lacy BE, Lembo AJ, Saito YA, Schiller LR, et al.American College of Gastroenterology monograph on the management of irritable bowel syndrome and chronic idiopathic constipation. Am J Gastroenterol. 2014;109 Suppl 1:S2-26.

15. World Gastroenterology Organisation. Irritable bowel syndrome: a global perspective (update September 2015) [Internet]. WGO; 2015. Available from: https://www.spg.pt/wp-content/uploads/2015/11/ 2015-IBS.pdf

16. McKenzie YA, Bowyer RK, Leach H, Gulia P, Horobin J, O'Sullivan NA, et al. British Dietetic Association systematic review and evidence-based practice guidelines for the dietary management of irritable bowel syndrome in adults (2016 update). J Hum Nutr Diet. 2016;29(5):549-75.

17. Canadian Association of Gastroenterology. Enhanced primary care pathway: irritable bowel syndrome [Internet]. Oakville: CAG; 2016. Available from: https://www.specialistlink.ca/files/IBS_Enhanced_Primary_Care_Pathway_Dec_2016_2.pdf

18. National Institute for Health and Care Excellence. Irritable bowel syndrome in adults: diagnosis and management. London: NICE; 2017. Available from: https://www.nice.org.uk/guidance/cg61/resources/irritablebowelsyndrome-in-adults-diagnosis-and-management-975562917829

19. Marsh A, Eslick EM, Eslick GD. Does a diet low in FODMAPs reduce symptoms associated with functional gastrointestinal disorders? A comprehensive systematic review and meta-analysis. Eur J Nutr. 2016;55(3):897-906.

20. Varjú P, Farkas N, Hegyi P, Garami A, Szabó I, Illés A, et al. Low fermentable oligosaccharides, disaccharides, monosaccharides and polyols (FODMAP) diet improves symptoms in adults suffering from irritable bowel syndrome (IBS) compared to standard IBS diet: a metaanalysis of clinical studies. PLoS One. 2017;12(8):e0182942.

21. Altobelli E, Del Negro V, Angeletti PM, Latella G. Low-FODMAP diet improves irritable bowel syndrome symptoms: a meta-analysis. Nutrients. 2017;9(9):940.

22. Rao SS, Yu S, Fedewa A. Systematic review: dietary fibre and FODMAPrestricted diet in the management of constipation and irritable bowel syndrome. Aliment Pharmacol Ther. 2015;41(12):1256-70.

23. Wong WM. Restriction of FODMAP in the management of bloating in irritable bowel syndrome. Singapore Med J. 2016;57(9):476-84.

24. Pedersen N, Andersen NN, Végh Z, Jensen L, Andersen DV, Felding M, et al. Ehealth: low FODMAP diet vs Lactobacillus rhamnosus GG in irritable bowel syndrome. World J Gastroenterol. 2014;20(43):16215-26.

25. Halmos EP, Power VA, Shepherd SJ, Gibson PR, Muir JG. A diet low in FODMAPs reduces symptoms of irritable bowel syndrome. Gastroenterology. 2014;146(1):67-75.e5.

26. Harvie R. A reduction in FODMAP intake correlates strongly with a reduction in IBS symptoms: the FIBS study [dissertation]. Dunedin, New Zealand: University of Otago; 2014. Available from: https://ourarchive.otago.ac.nz/handle/10523/4927 
27. Böhn L, Störsrud S, Liljebo T, Collin L, Lindfors P, Törnblom H, et al. Diet low in FODMAPs reduces symptoms of irritable bowel syndrome as well as traditional dietary advice: a randomized controlled trial. Gastroenterology. 2015;149(6):1399-407.e2.

\section{CONFLITO DE INTERESSES}

Os autores declaram não ter quaisquer conflitos de interesse.

\author{
ENDEREÇO PARA CORRESPONDÊNCIA \\ Joana Estrela Felício \\ E-mail: reisjoana18@gmail.com \\ https://orcid.org/0000-0001-8290-8642
}

Recebido em 07-12-2018

Aceite para publicação em 22-11-2019

\section{ABSTRACT \\ THE LOW-FODMAP DIET REDUCES SYMPTOMS OF IRRITABLE BOWEL SYNDROME? - AN EVIDENCE-BASED REVIEW}

Objective: We aimed to review the evidence for the efficacy of a low FODMAP (fermentable oligo-, di- and monosaccharides and polyols) diet in the management of gastrointestinal symptoms in irritable bowel syndrome (IBS).

Methods: We searched MEDLINE/PubMed, DARE, Trip DataBase, Evidence-based Medicine, Clinical Evidence, Web of Science, Canadian Medical Association Practice Guidelines, National Guidelines Clearing House and The Cochrane Library databases for guidelines, meta-analysis and systematic reviews published in English between 2009 and 2017, using the MeSH terms 'irritable bowel syndrome', 'signs and symptoms', 'diet therapy' and the non-MeSH term 'FODMAPs'. The Strength of Recommendation Taxonomy (SORT) scale was used to determine levels of evidence and strength of recommendations.

Results: Of 203 articles found, 11 studies were included: three meta-analyses, three systematic reviews, and five guidelines. There is evidence supporting the efficacy of a low FODMAP diet in improving gastrointestinal symptoms in IBS patients, mainly bloating and abdominal pain, especially in the diarrhea-predominant and mixed subtypes. Some studies show a greater improvement after six weeks. A low FODMAP diet seems to be at least as effective as traditional dietary advice for IBS. Some guidelines recommend this diet in the management of IBS patients, especially when traditional dietary advice is not effective. Conclusions: There is enough evidence to recommend a low FODMAP diet in the management of diarrhea-predominant and mixed subtypes IBS patients with bloating and abdominal pain (grade of recommendation B). There is no sufficient evidence on the long-term efficacy and safety of this diet.

Keywords: FODMAP; Irritable bowel syndrome; Gastrointestinal signs and symptoms; Diet therapy. 